\title{
An information integration approach to serial effects in verbal discrimination learning*
}

\author{
IRWIN P. LEVIN $\dagger$ and KENT L. NORMAN \\ University of Iowa, Iowa City, Iowa 52240
}

New experimental designs and analytic techniques are presented for investigating serial effects in verbal discrimination. These designs and techniques derive from earlier studies of serial information integration. On a given study trial, feedback is presented for a fixed percentage of the items. Across items in the list, feedback-nonfeedback sequences are generated by factorial designs that permit assessment of how the probability of a correct response on a given trial is influenced by feedback presentations on each preceding trial. The relative influence of each of the preceding feedback presentations is compared to provide an analysis of intertrial serial effects. Preliminary work showed nonmonotonic serial curves.

The delineation of serial effects is of primary importance in understanding the processing of information in verbal learning. This paper considers verbal learning as a form of serial information integration and views each reinforcing event as a source of information or feedback affecting choice behavior. This approach leads to new experimental designs and analytic techniques for examining the effect of each preceding reinforcing event on current response choice in tasks such as verbal discrimination.

As summarized by Anderson (1972), information integration theory has provided a useful context for examining serial integration processes in a variety of experimental tasks. A basic notion of the theory is that each piece of information in an integration task is represented by two parameters: a scale value, $s$, and a weight, w. In serial integration tasks, it is of particular interest to see how weight varies as a function of serial position. Most applications of the theory employ factorial designs for constructing stimulus sequences, where each stimulus value appears equally often at each serial position.

Despite the past success of information integration theory in serial integration tasks and despite its potential applicability to learning, recent reviews by Anderson (1972) and by Slovic \& Lichtenstein (1971) describe learning as a relatively unexplored area within the

*Requests for reprints should be sent to Irwin P. Levin, Department of Psychology, University of Iowa, Iowa City, Iowa 52242.

tThis paper was completed while the authors were visitors at the University of California, San Diego. The first author was supported by a Research Assignment from the University of lowa and by National Institute of Mental Health Grant MH23911-01, and the second author was supported by a United States Public Health Service research predoctoral fellowship, National Institute of Mental Health Grant MH-51134-01. Clerica assistance was provided through National Institute of Mental Health Grant MH-15828 awarded to the Center for Human Information Processing, University of California, San Diego. information integration approach. However, there have been a few studies dealing with an integration analysis of a particular type of learning task. Friedman, Carterette, \& Anderson (1968) and Levin, Dulberg, Dooley, \& Hinrichs (1972) applied an averaging model of information integration theory to two-choice probability learning. The model was shown to be a useful analytic tool for examining S's choice on a given trial as a function of the sequence of binary events on preceding trials.

The application of the experimental designs and analytic techniques of information integration theory can be extended to learning tasks such as verbal discrimination. Rather than varying the correct response over trials as in probability learning, the binary event for a given item on a given study trial is whether or not feedback is presented for that item. The response on a given test trial thus follows a sequence of feedback-nonfeedback presentations over preceding study trials. The structure of this sequence is varied across items in the list using factorial designs. This permits a quantitative analysis of how the probability of a correct response on a given trial is influenced by feedback presentations on preceding trials. While previous verbal learning studies have examined within-list serial position effects and between-trials response dependencies, there has been no previous work with verbal learning tasks to obtain serial curves of the type used here. The following is an illustration of this technique.

\section{EXPERIMENTAL PARADIGM AND METHODS OF ANALYSIS}

In a verbal discrimination task, study trials and test trials were alternated so that $S$ did not receive feedback for a particular test trial response until that item was presented on the next study trial. On each test trial, $S$ was to predict the correct response for each pair. The $S$ was told that one item was correct in each pair and that it remained correct on all trials. He was also told that the study trial following a given test trial presented feedback for only some of the pairs.

A list of 16 verbal discrimination pairs was presented on each test trial. Each study trial listed the correct response for eight of these pairs. Over four trials, each possible sequence of feedback (F) and nonfeedback $(N)$ presentations was represented by a different pair in the 16-pair list. This is shown in Table 1. A fifth test trial was presented, and performance on this trial was analyzed as a function of the presence vs absence of feedback on each of the preceding four study trials. 
Table 1

Sequences of Feedback (F) and Nonfeedback (N) Presentations Over Four Successive Study Trials and Response Proportions on Test Trial 5

\begin{tabular}{cccccc}
\hline & \multicolumn{2}{c}{ Study } & \multicolumn{2}{c}{ Trial Number } & \multicolumn{2}{c}{$\begin{array}{c}\text { Mean Proportion } \\
\text { Correct Responses } \\
\text { on Test Trial 5 }\end{array}$} \\
\cline { 2 - 5 } 1 & 1 & 2 & 3 & 4 & F \\
2 & F & F & F & F & .867 \\
3 & F & F & F & N & .822 \\
4 & F & F & N & F & .867 \\
5 & F & F & N & N & .800 \\
6 & F & N & F & F & .800 \\
7 & F & N & F & N & .800 \\
8 & F & N & N & F & .844 \\
9 & F & N & N & N & .756 \\
10 & N & F & F & F & .733 \\
11 & N & F & F & N & .756 \\
12 & N & F & N & F & .800 \\
13 & N & F & N & N & .689 \\
14 & N & N & F & F & .756 \\
15 & N & N & F & N & .511 \\
16 & N & N & N & F & .778 \\
Mean & Difference & for F & vs N & N & .333 \\
& .150 & .095 & .023 & .123 & \\
\hline
\end{tabular}

Since every possible feedback-nonfeedback sequence over four trials was presented, the experimental design can be categorized as a $2^{4}$ within-S complete factorial design.

The main effects of the $2^{4}$ design can be viewed rather simply. The effect of feedback on Study Trial 1 can be represented by the mean difference in number of correct responses on Test Trial 5 between those eight pairs for which feedback was given on Study Trial 1 (Pairs 1-8 in Table 1) and those eight pairs for which feedback was not given on Study Trial 1 (Pairs 9-16 in Table 1). In an analogous fashion, the effect of feedback on Study Trial 2 can be represented by the mean difference in number of correct responses on Test Trial 5 between those pairs for which feedback was presented on Study Trial 2 and those for which feedback was not presented on Study Trial 2, and similarly for the effects of feedback on Study Trials 3 and 4. Mean difference scores, converted to proportions by dividing by 8 , for a group of 45 Ss are shown at the bottom of Table 1 , and the mean proportion of correct responses for each sequence is shown at the right-hand side of Table 1 for these Ss. ${ }^{1}$ The mean difference scores can be compared in magnitude to yield a serial curve over successive study trials. In this case, it is U-shaped. For Study Trials 1, 2, and 4 , the means were significantly different from zero.

Similar analyses were conducted for performance on Test Trials 3 and 4 as a function of the presence vs absence of feedback on preceding study trials, but these analyses will not be included in detail here. Analyses of earlier test trials can be useful in understanding the nature of the serial effects. For example, in the present case, the effect of Study Trial 3, which was not significant in the analysis of performance on Test Trial 5, was highly significant in the analysis of Test
Trial 4. This suggests a transient recency effect.

It may be noted that the mean difference scores given in Table 1 are averaged over items with differing numbers of feedback presentations. Figure 1 illustrates a method of obtaining separate serial curves for items with one, two, and three feedback presentations. For items with a fixed total number of feedback presentations, Fig. 1 plots the mean proportion of correct responses for those items with feedback presented on Study Trial 1, those with feedback on Study Trial 2, and so forth. The separation of the curves shows that, as would be predicted by any learning theory, performance is directly related to the number of prior feedback presentations. The U-shaped relationship noted earlier is clearly evident for items with only one feedback presentation and is less evident for items with two or three feedback presentations where the curves are almost flat. This is not surprising, since the influence of serial factors would necessarily decrease as performance approached asymptote.

The interactions of the $2^{4}$ design can be used to test the assumption that the effects of feedback vs nonfeedback are independent across successive study trials. A greater-than-chance number of significant interactions leads to rejection of this assumption. In the present case, 3 out of 11 possible interactions were significant at the .05 level. These interactions were due to the fact that the influence of a given feedback presentation was inversely related to the number of prior feedback presentations for that item. Thus, for example, the largest difference between pairs of items which differed only in the presence vs absence of feedback on Study Trial 4 was for NNNF vs NNNN; and the next highest differences were for NNFF vs NNFN, NFNF vs NFNN, and FNNF vs FNNN. A parallel observation in Fig. 1 is that the separation of the curves for $1 F$ and $2 F$

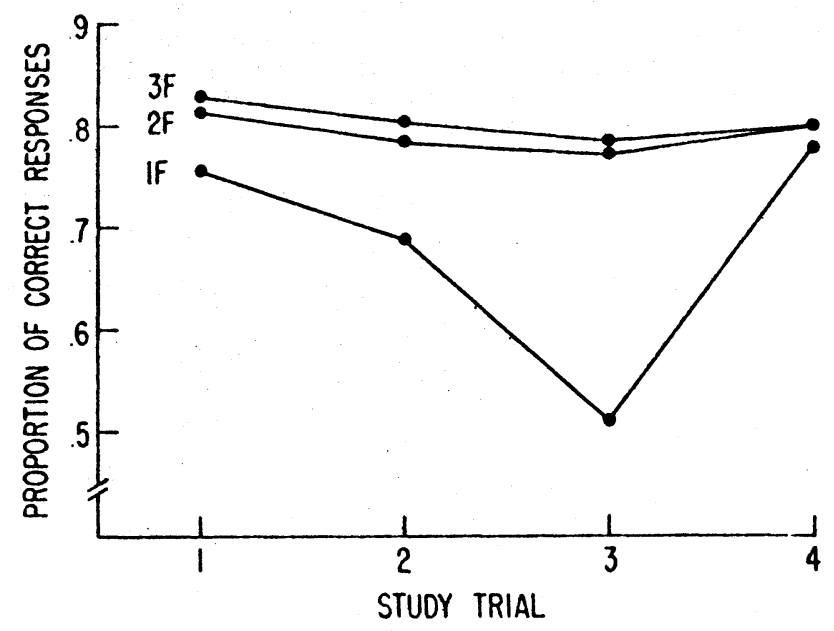

Fig. 1. Mean proportion of correct responses for those items with feedback presented on a given study trial. Separate serial curves are drawn for items with different total numbers of feedback presentations. 
is greater than the separation of the curves for $2 \mathrm{~F}$ and $3 \mathrm{~F}$. In terms of information integration constructs, it may be assumed that the weight or influence of a given feedback presentation has two nonadditive components, a serial component related to study trial number and an information-value component which diminishes with number of prior feedback presentations.

Further work is needed to solidify and interpret the findings presented here for verbal discrimination. In addition, the methodology described in this report may prove useful in a variety of learning tasks. Applications could include any outcome variable manipulated factorially over trials. The serial analyses illustrated here would show the extent to which the effects of these variables change over successive presentations.

\section{REFERENCES}

Anderson, N. H. Information integration theory: A brief survey.
Technical Report No. 24, Center for Human Information Processing, University of California, San Diego, April 1972.

Friedman, M. P., Carterette, E. C., \& Anderson, N. H. Long-term probability learning with a random schedule of reinforcement. Journal of Experimental Psychology, 1968, 78, 442-455.

Levin, I. P., Dulberg, C. S., Dooley, J. F., \& Hinrichs, J. V. Sequential dependencies in single-item and multiple-item probability learning. Journal of Experimental Psychology, $1972,93,262-267$.

Slovic, P., \& Lichtenstein, S. Comparison of Bayesian and regression approaches to the study of information processing in judgement. Organizational Behavior and Human Performance, 1971, 6, 649-744.

\section{NOTE}

1. Details of the procedure can be gotten by writing the first author. Briefly, on test trials, each of 16 pairs of low-frequency words was presented on a slide for 1.0 sec. During this interval, $S$ responded by pressing a button corresponding to the right-left position of his response choice. Position of a word within a pair and position of a pair within the list were varied over trials. On a study trial, the correct response for each of eight pairs was presented for $1.0 \mathrm{sec}$

(Received for publication March 22, 1973.)

\section{Cue utilization as a function of monetary incentive and learning efficiency*}

\author{
JEROME S. COHEN, GABOR A. TELEGDY \\ JEAN PAUL LAROCHE, and YAAKOV GETZ \\ University of Windsor, Windsor 11, Ontario, Canada
}

Different levels of monetary incentive, high (\$2.00), moderate $(\$ 0.50)$, or no reward, during either initial learning or testing conditions did not affect range of cue utilization in Ss required to learn a redundant relevant dimension concept task. Initial learning efficiency was related to cue utilization, however. Ss making more than three errors on the original task were generally unable to employ both relevant dimensions.

According to Easterbrook (1959), cue utilization of an organism is inversely related to drive level. This relationship has been found in human Ss under various types of drive conditions, such as general anxiety level (Zaffy \& Bruning, 1966), socially induced anxiety level (Bruning, Copage, Kozuh, Young, \& Young, 1968), and thirst and shock-induced fear (Solley, 1969). Bahrick (1954) found that high monetary incentive motivation (\$1.50 maximum) interfered with incidental learning of

*The present study was supported by funds from a National Research Council of Canada grant awarded to J. S. Cohen (A 7450). The authors wish to thank R. Daly and R. Orr of the Psychology Department, University of Windsor, and Z. Veres and the Windsor Public School Board for their help and cooperation. A modified version of the present study was read at the 1972 modified version of the present study was read at the 1972
Southeastern Psychological Association Convention in Atlanta, Georgia. a redundant color cue in a serial form anticipation task. The present study was an extension of Bahrick's (1954) original study on the effects of monetary incentives on cue utilization. Ss were required to master a concept classification task under one of three levels of reward, high ( $\$ 2.00$ maximum), moderate $(\$ 0.50$ maximum, and zero (no monetary reward). Each geometric figure could be classified in one of two categories by the use of one or both redundant relevant dimensions: figure closure and orientation of lines in figure. It was predicted that lower incentives would result in greater cue utilization, i.e., the use or recognition of both redundant relevant dimensions, than the high-incentive condition. Test procedures measuring cue utilization were carried out under counterbalanced incentive conditions in order to determine learning and performance effects of the various incentive conditions. The effect of incentive levels on dimension dominance was also investigated. An auxiliary investigation concerned the relationship between acquisition rate of the initial RRC task and cue utilization.

\section{METHOD}

Subjects

Sixty-three twelfth-grade boys from one of the public secondary schools in Windsor, Ontario, were used. During the 